\title{
How deep is too deep? Gastrointestinal complication of COVID-19 deep nasal swab testing
}

Deep nasal swab novel coronavirus (COVID-19) testing has become routine practice prior to hospitalization and almost all medical procedures since the start of the pandemic. We present the case of a 35-year-old woman at 40 weeks gestation who presented to the hospital for a scheduled elective induction and delivery for full-term pregnancy. On admission, she underwent routine testing for COVID-19 with deep nasal swab. The swab was inserted into her left naris, and while being rotated, broke off at the indicator line in the patient's posterior nasopharynx ( Fig. 1). Almost immediately, the patient felt pain in her throat that quickly subsided. Because the swab tip was lost, the patient underwent a second uneventful COVID-19 deep nasal swab, which was negative.

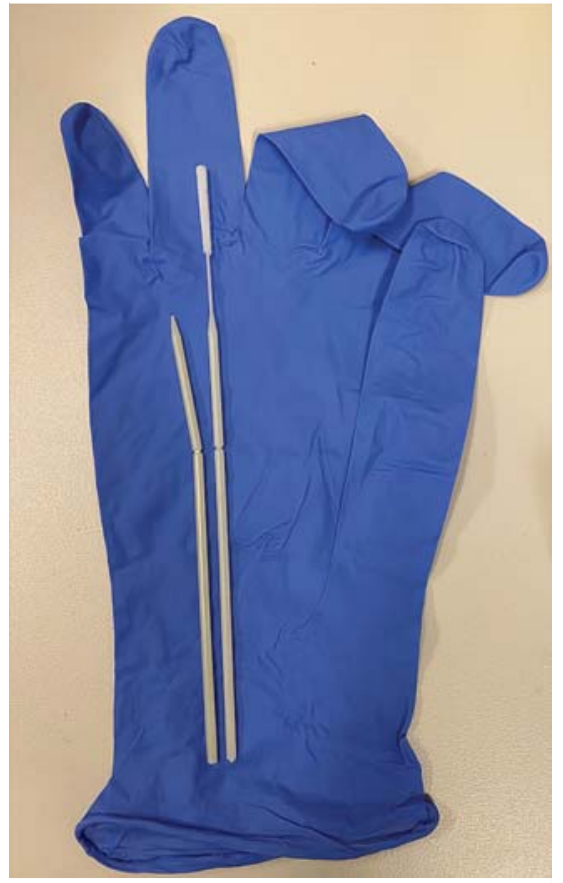

- Fig. 1 The COVID nasopharyngeal swab with broken tip from the patient compared to an intact COVID swab, with a medium-sized surgical glove in the background for comparison.
The patient's induced labor continued because the distal nasal swab tip was thought to be lodged in the nasopharynx. However, a stat $x$-ray and urgent laryngoscopy did not show the location of the swab. After experimenting by breaking similar COVID-19 swabs, it was determined that the broken plastic from the distal swab appeared to be sharp. Therefore, endoscopy was planned to locate and retrieve the swallowed swab tip. Following delivery of a healthy neonate, the patient underwent push enteroscopy 8 hours after suspected ingestion. Endoscopy demonstrated a normal esophagus, stomach, and proximal duodenum with no evidence of prior trauma. The broken swab was then located in the fourth portion of the duodenum ( $\triangleright$ Fig. 2a). Using raptor forceps, the swab tip was oriented vertically to safely grasp the sharp end ( Fig.2b). The swab tip was carefully removed with only mild gastric mucosal trauma on retrieval. The retrieved swab tip was compared to the broken swab shaft to confirm complete extraction.

COVID testing is standard practice prior to most routine medical procedures, however, rare complications have been reported [1]. To our knowledge, this is the third reported case of a swal- lowed COVID nasal swab and the only one retrieved from the small bowel [24]. Despite the fact that the broken swabs are $>4 \mathrm{~cm}$ long, the patient may not be aware of ingestion, as occurred in this case. Many deep nasal swabs have indicator lines that are designed to be broken after sampling before being sent for testing. When the swabs are broken at the indicator line, they are sharp and should be promptly retrieved. Trauma can be minimized by grasping the ingested swab tips from the sharp end for safe retrieval.

\section{Competing interests}

The authors declare that they have no conflict of interest.

\section{The authors}

\section{Domenico A. Farina, Kirbylee K. Nelson} Northwestern University, Feinberg School of Medicine, Division of Gastroenterology and Hepatology, Chicago, Illinois, United States
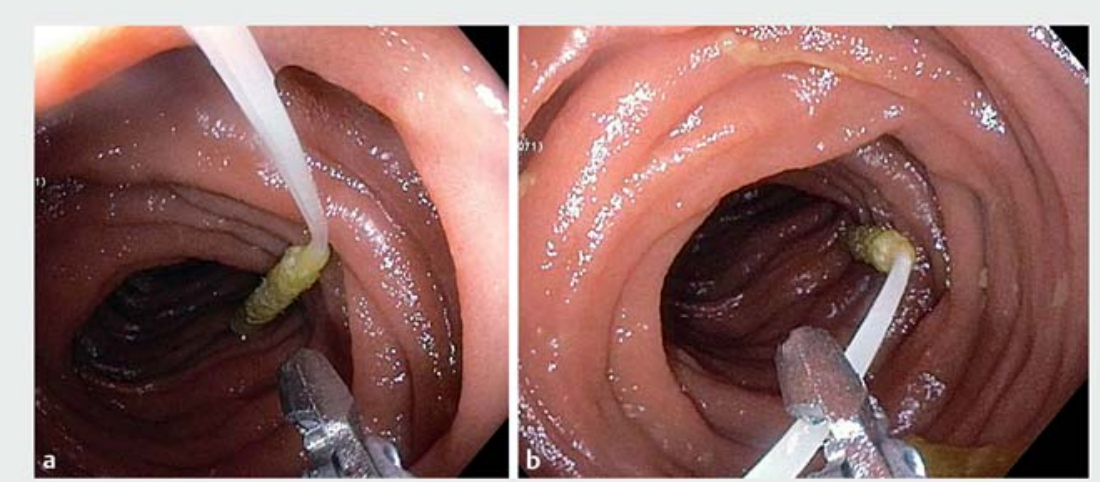

- Fig. 2 a The broken tip of the COVID nasopharyngeal swab in the fourth portion of the duodenum after orienting the sharp end toward the endoscope. $\mathbf{b}$ The sharp end of the broken COVID nasopharyngeal swab tip being grasped in preparation for extraction. 
Corresponding author

\section{Domenico A. Farina, MD}

Northwestern University, Feinberg School of Medicine, Division of Gastroenterology and Hepatology, 676 N St. Clair Street, Arkes Pavilion Suite 1400, Chicago, IL 60611

Fax: 312-695-3999

domenico.farina@northwestern.edu

\section{References}

[1] Föh B, Borsche M, Balck A et al. Complications of nasal and pharyngeal swabs - a relevant challenge of the COVID-19 pandemic? Euro Respiratory J 2021: 2004004 doi:10.1183/13993003.04004-2020
[2] De Luca L, Maltoni S. Is naso-pharyngeal swab always safe for SARS-CoV-2 testing? An unusual, accidental foreign body swallowing Clin J Gastroenterol 2021; 14: 4447

[3] Medas R, Coelho R, Macedo G. An unusual collateral damage of COVID-19 pandemic. Gastrointest Endosc 2020; 92: 1261-1262

[4] Gaffuri M, Capaccio P, Torretta S et al. An unusual retained choanal foreign body: a possible complication of COVID-19 testing with nasopharyngeal swab. Ear Nose Throat J 2021: doi:10.1177| 0145561321993933

\section{Bibliography}

Endosc Int Open 2021; 09: E1841-E1842

DOI 10.1055/a-1578-2273

ISSN 2364-3722

(c) 2021. The Author(s).

This is an open access article published by Thieme under the terms of the Creative Commons Attribution-NonDerivativeNonCommercial License, permitting copying and reproduction so long as the original work is given appropriate credit. Contents may not be used for commercial purposes, or adapted, remixed, transformed or built upon. (https:// creativecommons.org/licenses/by-nc-nd/4.0/)

Georg Thieme Verlag KG, Rüdigerstraße 14, 70469 Stuttgart, Germany 